\title{
Three-Dimensional Gesture Comparison Using Curvature Analysis of Position and Orientation
}

\author{
M.T. Pham, R. Moreau and P. Boulanger
}

\begin{abstract}
This paper describes a new analysis method dedicated to the comparison of human gestures. The orientations and the positions of the gestures are first digitized using active 3D sensors and then compared to a 6-D template using curvature analysis. The proposed algorithm first starts by computing the invariant curvature of $3 \mathrm{D}$ position and orientation of a surgical tool using Frenet-Serret frames in $3 \mathrm{D}$ and quaternion space. The resulting curvature calculation is matched and compared to the template using a Dynamic Time Warping method. The proposed method is invariant to sensor position and orientation. An experimental study shows the efficiency of the new algorithm for an application in obstetrics, where the aim is to compare forceps blade placements between a senior medical doctor and a novice.
\end{abstract}

\section{INTRODUCTION}

Several researchers have studied medical gestures in different fields: surgery, dermatology, orthopedics, and radiology for the design of medical robots or to improve surgical training techniques. Many methods relying on video analysis of gesture were proposed for laparoscopic tasks such as in Cao [1]. In those applications, gestures are decomposed into four main tasks (suturing, tying knots, cutting suture, and dissecting tissue). These tasks are then divided in several subtasks and the time needed to execute them is computed to be compared to an expert. A global rating scheme based on video analysis by an expert was developed in [2] to evaluate surgical performance. In some applications like obstetrics, it is not always possible to do video

M.T. Pham and R. Moreau are with the Ampere lab., INSA-Lyon, Université de Lyon, F-69621, France minh-tu. phameinsa-lyon. fr

P. Boulanger is with the AMMI lab., Dpt of Computing Science, University of Alberta, T6G2E8, Canada pierreb@cs.ualberta.ca analysis as most of those tasks are performed inside the maternal pelvis. In addition, the evaluations produced by those visual inspection techniques tend to be biased by the observer and are not completely objective.

The main objective of our approach is to create a metric that evaluate skill transfer and skill acquisition between an expert and trainee. Our gesture comparison method allows us to check qualitatively (by providing a performance index) the quality of a gesture compared to another one. In this paper, we will demonstrate that one can compare objectively the difference in gesture between an expert and novices in an invariant and time independent way. This paper is divided into three sections. Section 2 introduces the mathematical foundation of our method and explains its motivations. Section 3 is dedicated to an experimental validation. We then conclude by discussing the pros and cons of the current implementation and our future experimental plans.

\section{TRAJECTORY AND ORIENTATION IN 3D SPACE}

In this section, we present the mathematical foundation that describes trajectories of a point in $3 \mathrm{D}$ and orientation spaces. Using those notions one can localize objects in space not only in term of position but also in term of orientation.

\section{A. Notion of Space-Curve}

In differential geometry, one can represent a space curve as a set of points whose Cartesian coordinates $x, y$ and $z$ are continuous functions of a parameter $t$ varying inside an interval of $\mathbb{R}$. Mathematically this is expressed by:

$$
\mathbf{f}(t)=[x(t), y(t), z(t)]^{T}
$$




\section{B. Orientation Analysis}

Even though the concept of rotation is familiar, there are many ways to represent it mathematically, each with its own advantages and disadvantages. Generally, there are four main representations of rotation discussed in the literature: rotation matrix, axis-angle, Euler angle and quaternion [3], [4]. In this paper, we adopt quaternion for its is simplicity and its absence of singularities such as the gimbal lock [5].

A quaternion $\mathbf{q}$ consists of a scalar part $q_{w} \in \mathbb{R}$ and a vector part $\left(q_{x}, q_{y}, q_{z}\right)^{T} \in \mathbb{R}^{3}$.

$$
\mathbf{q}=\left[q_{w},\left(q_{x}, q_{y}, q_{z}\right)\right]^{T} .
$$

In practice, one can easily show that rotations could be represented by the sub-space of unit quaternion $\mathbb{H}_{1} \subset \mathbb{H}$ forming a unit sphere in a 4-dimensional space where:

$$
\|\mathbf{q}\|=q_{w}^{2}+q_{x}^{2}+q_{y}^{2}+q_{z}^{2}=1
$$

\section{Curvature in 3-dimensional Space}

As described by Reiger et al. [6], the curvature of a curve can completely describe the shape of this curve in 2D space. If one add a second parameter, the torsion, one can describe a curve in 3D space.

Lets define $\mathbf{r}(s)$ as a smooth trajectory in $\mathbb{R}^{3}$ parameterized by the arc length $s$. The vectors of the Frenet-Serret frame are an orthonormal basis iteratively constructed by applying the Gram-Schmidt process to the vectors $\mathbf{r}^{\prime}(s), \mathbf{r}^{\prime \prime}(s)$ and $\mathbf{r}^{(3)}(s)$, where $\mathbf{r}^{(i)}(s)(i=1, \ldots, 3)$ is the $\mathrm{i}^{\text {th }}$ of derivative of $\mathbf{r}(s)$ with respect to $s$. Applying the proprieties of the inner product and the collinearity of two multidimensional vectors, it is easy to demonstrate [7] that the formulas for the generalized curvature $k_{j}(j=$ $1,2)$ with respect to the arc length parametrization can be express as:

$$
\begin{aligned}
& k_{1}=\left\|\mathbf{r}^{\prime} \wedge \mathbf{r}^{\prime \prime}\right\|=\left\|\mathbf{r}^{\prime \prime}\right\| \\
& k_{2}=\frac{\left\|\mathbf{r}^{\prime} \wedge \mathbf{r}^{\prime \prime} \wedge \mathbf{r}^{\prime \prime \prime}\right\|}{\left\|\mathbf{r}^{\prime} \wedge \mathbf{r}^{\prime \prime}\right\|^{2}}
\end{aligned}
$$

where $\wedge$ denotes the outer product for multidimensional vectors defined by as in Grassmann's algebra [8].

\section{Dynamic Time Warping}

One contribution of this paper is to reduce the order of the problem of 3D trajectories comparison to a 1D case by using a Dynamic Time Warping (DTW) [9] algorithm to find the alignment of the different curvature vectors instead of trying to directly align multidimensional time-series such as 3D-positions [10]. Using DTW one can find the optimal alignment in the sense that it minimizes the sum of the local distances $d(i, j)$ between the aligned observation pairs $\left(A_{i}, B_{j}\right)$ of two sequences $\mathbf{A}$ and $\mathbf{B}$. The distance metric $d(i, j)$ between the two signals is defined as the square of the Euclidean distance:

$$
d(i, j)=\left(A_{i}-B_{j}\right)^{2}
$$

\section{E. Performance Index}

In order to provide a full description of the position and the orientation for a gesture, one can compute the curvature of the trajectory in 3-D space and and its associated quaternion (4) trajectory in 4D space. For orientation, one needs to keep in mind that only three of the four components of the quaternion curve (4) are independent, the last variable can be deduced from the other one, up to a sign, from (5). This means that it is possible to reduce the dimensionality of the problem by one for the orientation analysis. This implies that the curvature computation leads to a 3-dimensional problem for the position as well as for the orientation.

From the curvature and torsion calculation of the position and quaternion one can then use a Dynamic Time Warping method (section II-D) to find the alignment of the different curvature vectors. Finally the Pearson's product-moment correlation is computed to measure of the dependence between the reference template and the manipulations. The correlation coefficients $r_{p r}$ between a curvature vector $\mathbf{A}$ and a curvature vector $\mathbf{B}$ is given by:

$$
r_{p r}=\frac{\sum_{i=1}^{i=n}\left(\mathbf{A}_{i}-\overline{\mathbf{A}_{m}}\right)\left(\mathbf{B}_{i}-\overline{\mathbf{B}_{m}}\right)}{\sqrt{\sum_{i=1}^{i=n}\left(\mathbf{A}_{i}-\overline{\mathbf{A}_{m}}\right)^{2} \sum_{i=1}^{i=n}\left(\mathbf{B}_{i}-\overline{\mathbf{B}_{m}}\right)^{2}}}
$$

with :

$n$ is the size of the curvature vectors $\mathbf{A}$ and $\mathbf{B}$; 
$\mathbf{A}_{i}$ is the $i^{t h}$ component of the first curvature vector; $\overline{\mathbf{A}_{m}}$ is the mean of the components of $\mathbf{A}$;

$\mathbf{B}_{i}$ is the $i^{\text {th }}$ component of the second curvature vector;

$\overline{\mathbf{B}_{m}}$ is the mean of the components of $\mathbf{B}$.

\section{RESULTS}

A childbirth simulator was developed in our laboratory to train residents to place forceps blade [11]. This simulator consists of a realistic manikin to reproduce accurately the maternal pelvis and the fetal head. A forceps has been instrumented with two (one in each blade) electromagnetic sensors in order to measure its six degrees of freedom (dof), the 3-D position $[x, y, z]^{T}$ and the 3 angles $[\varphi, \alpha, \psi]^{T}$ (roll, pitch, yaw). Using these 6 dof sensors, it is possible to record forceps blade paths to be analyzed and compared with the trajectories of an expert. The simulator training is supervised under the authority of an expert obstetrician who is the class instructor. An expert obstetrician is defined as an obstetrician with at least ten years of experience, and who have used forceps in more than $80 \%$ of his instrumental interventions. The study protocol is the following. Five students were asked to participate in two 60 minutes sessions. Because this study is performed on inanimate simulator no ethic committee approbation is necessary. During the experiment, trainees were asked to perform a clinical examination and then perform 30 forceps blade placements. The forceps placements are performed during two sessions of 15 forceps blade placements with a seven days gap. Forceps placement is performed by sets of five, then the recorded trajectories is displayed on the screen, discussed with the teacher and a new set is performed. The experiment is stopped after 30 forceps placement providing for each trainee 60 trajectories: 30 left blade trajectories and 30 right blade trajectories. For every experiment, the fetal head is positioned according to the ACOG (American College of Obstetrics and Gynecology) classification [12] on an outlet $\mathrm{OA}+4$ presentation (Occiput Anterior location and station $+4 \mathrm{~cm}$ from the ischial spines plan) and exhibited no asynclitism, caput succedaneum or modeling mimicking a rather simple forceps blade instrumented delivery. Location OA means that forceps need be placed in a symmetrical way, both blades will have symmetrical path. During the training, the trainees are not aware of those information.

In practice, the data acquisition system used during the experiments, only allowed us to measure the curvature $\left(k_{1}\right)$ and not the torsion $\left(k_{2}\right)$. This implies that the computed curvature correlation coefficients are only a necessary but not sufficient parameter to perform full gesture comparison. In the following study, we compute the curvature's correlation coefficient (8) to determine the improvement score of a novice relative to the expert.

\section{A. Position and Orientation Comparison}

As explained in Section II-E, one can compute the correlation coefficients between the curvature signals of the expert and the curvature signals between the novices and the expert. Given an expert's attempt, we calculated the correlation coefficient with all the 4 attempts one by one by means of the method previously described in Section IIE. We gave the mean correlation coefficient and its standard deviation (in the parentheses) as a similarity metric in Table I. The computation of the novices' performance indexes for the position curve and the quaternion curve are given in table II and in table III, respectively. The results clearly show that there is a difference between the novice and the expert (Table I) in terms not only of forceps placement but also of forceps orientation.

TABLE I

EXPERT'S PERFORMANCE INDEX: CURVATURE OF THE POSITION AND QUATERNION TRAJECTORIES

\begin{tabular}{|c|c||c||c||}
\hline \multicolumn{2}{|c||}{$r_{p r}$} & position & quaternion \\
\hline \hline \multirow{2}{*}{$\begin{array}{l}\text { Expert } \\
\text { indeex }\end{array}$} & LFB & $88 \%(0.01)$ & $81 \%(0.06)$ \\
\cline { 2 - 4 } & RFB & $86 \%(0.05)$ & $89 \%(0.07)$ \\
\hline \hline
\end{tabular}

Except for trainee 4, three trainees manage to improve their performance indexes from the first day to the second day. At the end of the training, the trainee 5 obtained similar results than the first day. All these results can qualitatively be obtained by the visualizing either on the $3 \mathrm{D}$ position trajectories 
TABLE II

PERFORMANCE INDEX: CURVATURE OF THE POSITION

TRAJECTORY

\begin{tabular}{|c|c||c||c||}
\hline \multicolumn{2}{|c||}{$r_{p r}$} & Day I & Day II \\
\hline \hline Novice & LFB & $80 \%(0.09)$ & $80 \%(0.07)$ \\
\cline { 2 - 4 } 1 & RFB & $65 \%(0.08)$ & $87 \%(0.07)$ \\
\hline \hline Novice & LFB & $82 \%(0.07)$ & $82 \%(0.08)$ \\
\cline { 2 - 4 } 2 & RFB & $66 \%(0.09)$ & $73 \%(0.10)$ \\
\hline \hline Novice & LFB & $85 \%(0.05)$ & $81 \%(0.09)$ \\
\cline { 2 - 4 } 3 & RFB & $72 \%(0.08)$ & $83 \%(0.08)$ \\
\hline \hline Novice & LFB & $89 \%(0.04)$ & $71 \%(0.05)$ \\
\cline { 2 - 4 } 4 & RFB & $82 \%(0.07)$ & $62 \%(0.09)$ \\
\hline \hline Novice & LFB & $80 \%(0.07)$ & $76 \%(0.08)$ \\
\cline { 2 - 4 } 5 & RFB & $69 \%(0.12)$ & $72 \%(0.07)$ \\
\hline \hline
\end{tabular}

TABLE III

PERFORMANCE INDEX: CURVATURE OF THE QUATERNION TRAJECTORY

\begin{tabular}{|c|c||c||c||}
\hline \multicolumn{2}{|c||}{$r_{p r}$} & Day I & Day II \\
\hline \hline \multirow{2}{*}{$\begin{array}{c}\text { Novice } \\
1\end{array}$} & LFB & $73 \%(0.12)$ & $80 \%(0.06)$ \\
\cline { 2 - 4 } & RFB & $67 \%(0.07)$ & $87 \%(0.09)$ \\
\hline \hline \multirow{2}{*}{ Novice } & LFB & $76 \%(0.14)$ & $82 \%(0.09)$ \\
\cline { 2 - 4 } 2 & RFB & $64 \%(0.10)$ & $76 \%(0.07)$ \\
\hline \hline \multirow{2}{*}{ Novice } & LFB & $67 \%(0.11)$ & $83 \%(0.08)$ \\
\cline { 2 - 4 } 3 & RFB & $70 \%(0.08)$ & $77 \%(0.08)$ \\
\hline \hline \multirow{2}{*}{ Novice } & LFB & $74 \%(0.12)$ & $81 \%(0.10)$ \\
\cline { 2 - 4 } 4 & RFB & $71 \%(0.10)$ & $68 \%(0.07)$ \\
\hline \hline Novice & LFB & $75 \%(0.09)$ & $77 \%(0.11)$ \\
\cline { 2 - 4 } 5 & RFB & $77 \%(0.11)$ & $80 \%(0.07)$ \\
\hline \hline
\end{tabular}

or on the orientation trajectories in the quaternion space.

\section{CONCLUSION}

Objective operator evaluation is critical for the validation of training method in various fields such as medicine. In this paper, a general purpose method based on the six degree of freedom measurements of a medical gesture was developed that allowed us to quantify and to compare the differences between two operators in an invariant manner. By calculating the curvature during a manipulation, it is possible to evaluate the quality of the gesture performed by a novice compared to an expert. The proposed method allows us to analyze the orientations of the gesture by using a quaternion representation. We have shown that from a theoretical viewpoint the problem for $3 \mathrm{D}$ gesture comparison leads us to the computation of the curvature and the torsion of two 3-dimensional trajectories (position and quaternion). Instead of trying to align multidimensional 3D-positions, a DTW algorithm is used on a 1D signal instead.

\section{REFERENCES}

[1] C. Cao, C. MacKenzie, and S. Payandeh, "Task and motion analyses in endoscopic surgery," in ASME IMECE Symposium on haptic interfaces for virtual environement and teleoperator system, 1996, pp. 583-590.

[2] V. Datta, S. Bann, M. Mandalia, and A. Darzi, "The surgical efficiency score: a feasible, reliable, and valid method of skills assessment," The American Journal of Surgery, vol. 192, no. 3, pp. 372-378, 2006.

[3] W. Hamilton, Elements of Quaternions, C. University Press, Ed. Cambridge University Press, 1899.

[4] A. Hanson, Visualizing Quaternions, T. M. K. S. in Interactive 3D Technology, Ed. The Morgan Kaufmann Series in Interactive 3D Technology, 2006.

[5] J. Kuipers, Quaternions and Rotation Sequences, P. U. Press, Ed. New Jersey: Princeton University Press, 1999.

[6] B. Rieger and L. van Vliet, "Curvature of n-dimensional space curves in grey-value images," IEEE Transactions on Image Processing, vol. 11, no. 7, pp. 738-745, July 2002.

[7] Y. Aminov, Differential Geometry and Topology of Curves, Gordon and B. S. Publishers, Eds. Gordon and Breach Science Publishers, 2000.

[8] H. Grassmann, A New Branch of Mathematics: The Ausdenungslehre of 1844, O. C. P. Co, Ed. Open Court Publishing Co, 1994.

[9] L. Rabiner and B. Juang, Fundamentals of Speech Processing, Prentice-Hall, Ed. New Jersey: Prentice-Hall, 1993.

[10] M. Vlachos, M. Hadjieleftheriou, D. Gunopulos, and E. Keogh, "Indexing multidimensional time-series," The VLDB Journal, vol. 15, no. 1, pp. 1-20, 2006.

[11] R. Moreau, M. Pham, R. Silveira, T. Redarce, X. Brun, and O. Dupuis, "Design of a new instrumented forceps: application to a safe obstetrical forceps blade placement," IEEE Transactions on Biomedical Engineering, vol. 54, no. 7, pp. 1280-1290, July 2007.

[12] G. Cunningham, L. Gilstrap, K. Leveno, S. Bloom, J. Hauth, and K. Wenstrom, Williams Obstetrics, 22nd ed. the McGraw-Hill Companies, 2005, iSBN 0071413154. 